\title{
Phylogenetic networks that are their own fold-ups
}

\author{
K.T. Huber ${ }^{\mathrm{a}, *}$, G.E. Scholz ${ }^{\mathrm{b}}$ \\ ${ }^{a}$ School of Computing Sciences, University of East Anglia, Norwich, UK. \\ ${ }^{b}$ LIRMM, Université de Montpellier, CNRS, Montpellier, France.
}

\begin{abstract}
Phylogenetic networks are becoming of increasing interest to evolutionary biologists due to their ability to capture complex non-treelike evolutionary processes. From a combinatorial point of view, such networks are certain types of rooted directed acyclic graphs whose leaves are labelled by, for example, species. A number of mathematically interesting classes of phylogenetic networks are known. These include the biologically relevant class of stable phylogenetic networks whose members are defined via certain "fold-up" and "un-fold" operations that link them with concepts arising within the theory of, for example, graph fibrations. Despite this exciting link, the structural complexity of stable phylogenetic networks is still relatively poorly understood. Employing the popular tree-based, reticulation-visible, and tree-child properties which allow one to gauge this complexity in one way or another, we provide novel characterizations for when a stable phylogenetic network satisfies either one of these three properties.
\end{abstract}

Keywords: phylogenetic network, stable, tree-based, tree-child, reticulation-visible

2000 MSC: 05C05, 05C20, 05C85, 05D15, 92D15

\section{Introduction}

Phylogenetic networks are becoming of increasing interest to evolutionary biologists due to their ability to capture complex non-treelike evolutionary processes. Reflecting to some extent the different evolutionary contexts within which such processes can arise has led to the introduction of a number of mathematically interesting classes of such structures [12, 22, 27]. These include the class of stable phylogenetic networks which have already proven useful for better understanding how, for example, polyploidy arose (see e.g. 4, 20, 24]).

From a combinatorial point of view, a phylogenetic network is essentially a rooted directed acyclic graph whose set of leaves, that is, vertices of indegree one and outdegree zero, is labelled

\footnotetext{
*Corresponding author
} 
by a pre-given set $X$ of, for example, species. Now a phylogenetic network $N$ is called stable if it can be thought of as the "fold-up" of a certain multi-labelled tree $\mathcal{U}(N)$ into which a phylogenetic network $N$ can be "unfolded'] Sometimes just called $M U L$-trees [6, 15, 16, 18, such trees may be viewed as the phylogenetic analogue of the universal cover of a digraph [19] and differ from the type of phylogenetic trees commonly used by evolutionary biologist by allowing the leaf set to be a multi-set rather than just a set. For example, for the phylogenetic network $N$ depicted in terms of solid lines in Figure 1(i), we picture the tree $\mathcal{U}(N)$ in terms of solid lines in Figure 1(ii)

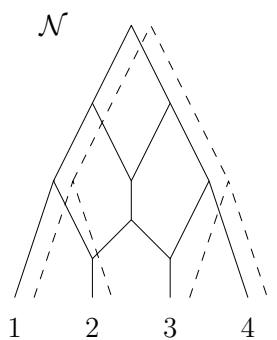

(i)

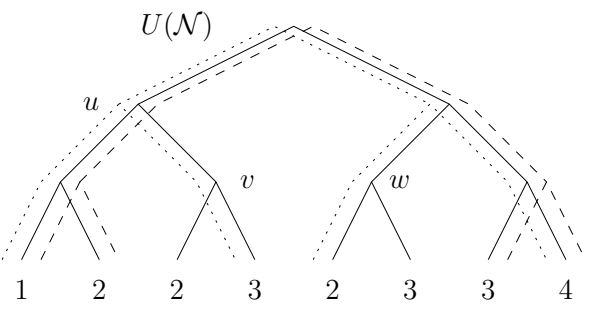

(ii)

Figure 1: (i) A phylogenetic network $N$ on $X=\{1,2,3,4\}$ that is stable (solid lines). (ii) Ignoring the interior labels for the moment, the MUL-tree $\mathcal{U}(N)$ obtained by unfolding $N$ (solid lines). Deferring the precise definitions to the next sections, we indicate in (i) a phylogenetic tree $T$ on $X$ in dashed lines that is displayed by $N$. In addition, we indicate in (ii) one of the ways $T$ is endorsed by $\mathcal{U}(N)$. Note that the phylogenetic tree on $X$ given in dotted lines in (ii) is endorsed by $\mathcal{U}(N)$ but not displayed by $N$.

- see the next section for a brief review of both operations and e.g. [19] for recent results linking the un-fold and fold-up operations to concepts arising in the theory of graph fibrations and also to the gene tree/species tree reconciliation problem [2, 30]. Despite these encouraging results, it is however still largely unclear how structurally complex a stable phylogenetic network can be.

To help illustrate this question which lies in the center of this paper, consider again the phylogenetic network $N$ depicted in Figure 1(i). Then $N$ is relatively simple in the sense that it is "tree-based" [7, 8, 14, 25, 31, that is, it can be thought of as a rooted tree $T$ with leaf set $\{1,2,3,4\}$ (called the base tree) to which arcs joining distinct arcs in $T$ have been added. As was shown in, for example, [23] not every stable phylogenetic network need however be treebased. On the other hand, if the complexity of $N$ is based on the notion of "reticulation-visible" 11, 11, 29] (essentially, this means that for every vertex $h$ in a phylogenetic network $N^{\prime}$ with indegree two or more there exists a leaf $x_{h}$ such that $h$ lies on every directed path from the root

\footnotetext{
${ }^{1}$ Note that the notions of a "stable phylogenetic network" and a "nearly stable phylogenetic network" [9] are unrelated - see Section 6 for more on this.
} 
of $N^{\prime}$ to $x_{h}$ ) then $N$ is not simple as the highest up vertex of $N$ with indegree two does not satisfy that property. Denoting that vertex by $h$ it is clear however that $N$ can easily be turned into a reticulation-visible network on $\{1, \ldots, 5\}$ by subdividing the outgoing arc of $h$ by a new vertex $x$ and adding the arc $(x, 5)$ and a leaf labelled "5". Since the resulting network is still stable, it follows that there exist stable phylogenetic networks (that are not also phylogenetic trees!) that are reticulation-visible. To help establish our results which include characterizations of stable phylogenetic networks that are tree-based or reticulation-visible, we employ numerous maps between the vertex sets of various graphs of interest to us, the most important of which is a map $\overline{\xi_{C}^{+}}$whose definition we defer to Section 4 as it is quite involved. For the convenience of the reader, we summarize them in terms of a commutative diagram in Figure 4

The outline of the paper is as follows. In the next section we collect relevant basic definitions. In addition, we review the aforementioned un-fold and fold-up operations for phylogenetic networks which underpin the definition of a stable phylogenetic network. Focusing on such networks, we recall in Section 4 the definition of a phylogenetic tree being displayed by a phylogenetic network. Subsequent to this, we characterize when such a network displays a phylogenetic tree in terms of when our map $\overline{\xi_{C}^{+}}$is injective (Theorem 4.2 . Using this insight, we then turn our attention to understanding three distinct popular properties of phylogenetic networks in terms of properties of $\overline{\xi_{C}^{+}}$. These are the aforementioned tree-based and reticulation-visible properties and the popular "tree-child" property [3, 5]. The latter essentially means that for every non-leaf vertex of a phylogenetic network at least one of its children has indegree one. In particular, in Section 5 we characterize when a phylogenetic tree is a base tree of a stable phylogenetic network in terms of that map (Theorem 5.1) and in Section 6, we characterize when such networks are tree-child or reticulation-visible in terms of the map $\overline{\xi_{C}^{+}}$(Theorem 6.4. - We refer the reader to [27. Figure 10.12] for a visualization of the interrelationships between network properties such as the ones of interest to us for a certain type of phylogenetic network. In Section 7 we turn our attention to the problem of characterizing stable phylogenetic networks in terms of combinatorial structures other than MUL-trees. We conclude with Section 8 where we outline future directions of research which might be of interest to pursue.

\section{Preliminaries}

In this section, we present relevant basic definitions. For this, we assume from now on that $X$ is a finite set of size at least three, unless stated otherwise. We start with some basic concepts from graph theory. 


\subsection{Graphs and DAGs}

Suppose $G$ is a rooted directed connected graph with vertex set $V(G)$ and arc set $A(G)$ that may or may not contain parallel arcs. Then we denote by $\rho_{G}$ the unique root of $G$. If $a \in A(G)$ is an $\operatorname{arc}$ of $G$ then we denote by $\operatorname{tail}(a)$ the start vertex of $a$ and by $\operatorname{head}(a)$ the end vertex of $a$. Furthermore, we write $(x, y)$ for an arc $a$ with $x=\operatorname{tail}(a)$ and $y=\operatorname{head}(a)$. If $G$ does not contain any directed cycles then we call it a rooted directed acyclic pseudo-graph, or rooted connected pseudoDAG, for short. A rooted connected pseudoDAG that does not contain any parallel arcs is called a rooted directed acyclic graph, or rooted connected DAG, for short. We refer to the replacement of an arc $a$ of $G$ by the directed path $\operatorname{tail}(a), w, \operatorname{head}(a)$ where $w$ is a new vertex not contained in $V(G)$ as a subdivision of $a$. In this case we also call $w$ a subdivision vertex of $a$. A (pseudo)graph obtained from $G$ via a succession of arc-subdivision operations is called a subdivision of $G$.

Suppose for the following that $G$ is a rooted connected pseudoDAG and that $v$ is a vertex of $G$. Then we call the number of incoming arcs of $v$ the indegree of $v$, denoted by indeg $(v)$, and its number of outgoing arcs the outdegree of $v$, denoted by outdeg $(v)$. We call a vertex with outdegree zero a leaf of $G$ and a vertex of $G$ that is not a leaf an interior vertex of $G$. The set of all leaves of $G$ is denoted by $L(G)$ and the set of all interior vertices of $G$ is denoted by $V^{\circ}(G)$. Moreover, we call a vertex $v$ of $G$ a tree vertex if $\operatorname{indeg}(v) \leq 1$, and a hybrid vertex of $G$ if $\operatorname{indeg}(v) \geq 2$. We denote the set of tree vertices of $G$ by $V^{-}(G)$.

We say that a vertex $w$ of $G$ is below a vertex $v$ of $G$ if there exists a directed path from $v$ to $w$ (note that a vertex $v$ is below itself). If, in addition, $v \neq w$ then we say that $w$ is strictly below $v$. If $w$ is below $v$ then we call $v$ an ancestor of $w$. In case $G$ is a tree, we call for any set $Y \subseteq L(G)$ of size two or more, the unique last vertex of $G$ that simultaneously lies on every directed path from $\rho_{G}$ to an element in $Y$ the last common ancestor of the elements of $Y$. We denote this vertex by $l c a_{G}(Y)$ (where we omit the index $G$ if the graph considered is clear from the context).

\subsection{Phylogenetic networks and MUL-trees}

Inspired by [19], we define a $X$-network $N=(N, \nu)$ to be an ordered pair consisting of a rooted connected pseudoDAG $N$ such that $\nu: X \rightarrow L(N)$ is a bijective map from $X$ into the leaf set of $N$ and (i) the root $\rho_{N}$ of $N$ has indegree zero, (ii) every non-leaf tree vertex has outdegree two, (iii) every hybrid vertex has outdegree one, and (iv) all leaves have degree one. To help keep notation at bay, we always assume that the leaf set of an $X$-network $\mathcal{N}=(N, \nu)$ is 
in fact $X$ (implying that $\nu$ is the identity map on $X$ ). In case there is no confusion, we therefore denote an $X$-network $\mathcal{N}=(N, \nu)$ simply by $N$. If $N$ is in fact a DAG and therefore does not

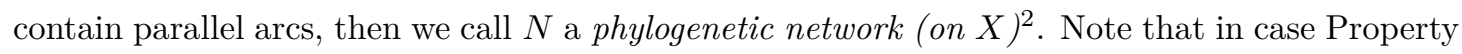
(iii) is strengthened by requiring that every hybrid vertex has indegree two then $N$ is called a binary phylogenetic network. Note that a phylogenetic network on $X$ that does not contain a hybrid vertex is generally called a phylogenetic tree (on $X$ ) (see e. g. [26]). Also note that since every non-leaf tree vertex of a phylogenetic network (on $X$ ) has outdegree two it follows that a phylogenetic tree (on $X$ ) must be binary. A phylogenetic tree $T$ on $X=\{a, b, c\}$ is called a triplet (on $X$ ). If $l c a_{T}(\{a, b\})$ is strictly below $l c a_{T}(\{a, c\})$ then we denote $T$ by $a b \mid c$.

Motivated by the definition of a phylogenetic tree on $X$, we define for $|X| \geq 1$ the MUL-tree $\mathcal{M}$ on $(X)$ to be an ordered pair $(M, \mu)$ where $M$ is a rooted tree for which $\operatorname{indeg}\left(\rho_{M}\right)=0$ holds and no vertex has indegree and outdegree one, and $\mu: X \rightarrow \mathcal{P}(L(M))$ is a labelling map from $X$ into the set $\mathcal{P}(L(M))$ of all non-empty subsets of the leaf set $L(M)$ of $M$ such that the following properties are satisfied: (i) For every leaf $l \in L(M)$ there exists some $x \in X$ with $l \in \mu(x)$, and (ii) there exists no leaf $l \in L(M)$ and $x, x^{\prime} \in X$ distinct such that $l \in \mu(x) \cap \mu\left(x^{\prime}\right)$ - see Figure 1(ii) for an example of such a tree for $X=\{1,2,3,4\}$. Informally speaking, Properties (i) and (ii) state that every leaf of $M$ must be labelled by precisely one element in $X$. Note that if, for all $x \in X$, the size of $\mu(x)$ is one and $|X| \geq 3$ then we implicitly identify $\mu(x)$ with its unique element, thus rendering $M$ a phylogenetic tree on $X$ in case $M$ is binary.

Suppose for the following that $\mathcal{M}=(M, \mu)$ is a MUL-tree on $X$. If $\mathcal{M}^{\prime}=\left(M^{\prime}, \mu^{\prime}\right)$ is a further MUL-tree on $X$ then we say that $\mathcal{M}$ and $\mathcal{M}^{\prime}$ are isomorphic if there exists a bijection $\eta: V(M) \rightarrow V\left(M^{\prime}\right)$ that induces a graph isomorphism between $M$ and $M^{\prime}$ such that $\mu^{\prime}(x)=$ $\{\eta(y): y \in \mu(x)\}$ holds for all $x \in X$. For a vertex $v \in V(M)$ that is not the root of $\mathcal{M}$ we denote by $\mathcal{M}_{v}=\left(M_{v}, \mu_{v}\right)$ the MUL-tree with root $v$ obtained from $\mathcal{M}$ by deleting the incoming arc of $v$ in $M$ and, to obtain $\mu_{v}$, restricting $\mu$ to the subset of $X$ that contains the leaf labels of the set of leaves of $M$ below $v$. We call a MUL-tree $\mathcal{M}^{\prime}=\left(M^{\prime}, \mu\right)$ a subMUL-tree of $\mathcal{M}$ if $\mathcal{M}^{\prime}$ is either $\mathcal{M}$ itself or there exists some $v \in V(M)$ such that $\mathcal{M}^{\prime}$ and $\mathcal{M}_{v}$ are isomorphic.

To help keep notation at bay, we also denote from now on the vertex set and arc set of a MUL-tree $\mathcal{M}$ by $V(\mathcal{M})$ and $A(\mathcal{M})$, respectively.

\footnotetext{
${ }^{2}$ Phylogenetic networks that enjoy Property (ii) were called semi-resolved phylogenetic networks in [19].
} 


\section{Stable phylogenetic networks}

In this section, we present a formal definition of a stable phylogenetic network. Since this definition relies on a certain "un-fold" operation for phylogenetic networks to obtain a MUL-tree and a certain "fold-up" operation for MUL-trees to obtain an X-network, we briefly review these constructions first. For details on both of them, we also refer the interested reader to [16] (see also [19]).

\subsection{The un-fold of a phylogenetic network}

To be able to outline this construction, we require further notation. Suppose $N$ is a phylogenetic network on $X$. The set of all directed paths of $N$ starting at $\rho_{N}$ and ending in a vertex of $V(N)$ is denoted by $\pi(N)$. For an element $x \in X$, we denote by $\pi_{x}(N)$ the subset of directed paths of $\pi(N)$ that end in $x$. Finally, we put $\pi_{0}(N)=\bigcup_{x \in X} \pi_{x}(N)$.

Viewable as a three-step process, the construction of the un-fold $\mathcal{U}(N)=\left(U(N), \phi_{N}\right)$ of $N$ works as follows. In the first step, we construct a tree $U^{*}(N)$ from $N$ whose vertices are the elements in $\pi(N)$ and whose arcs are the pairs $\left(P, P^{\prime}\right)$ in $\pi(N) \times \pi(N)$ for which there exists an arc $a \in A(N)$ such that $\operatorname{tail}(a)$ is the end vertex of $P$, the end vertex of $P^{\prime}$ equals head(a), and $P^{\prime}$ is the path $P$ extended by the arc $a$. To obtain the tree $U(N)$, the vertices of $U^{*}(N)$ with indegree and outdegree one are then suppressed, where by suppressing such a vertex $v$, we mean deleting $v$ as well as the incoming and outgoing arcs of $v$, and adding an arc from the parent of $v$ to the child of $v$. Observe that the suppressed vertices of $U^{*}(N)$ are precisely the paths in $\pi(N)$ ending at a hybrid vertex of $N[19]$.

The next and final step of the construction of $\mathcal{U}(N)$ is concerned with defining the map $\phi_{N}: X \rightarrow \mathcal{P}\left(L\left(U^{\prime}\right)\right)$ where we put $U^{\prime}=U(N)$. Since, as was observed above, hybrid vertices are the only vertices of $N$ with a single outgoing arc, there exists a bijection $\Psi_{N}: \pi^{-}(N) \rightarrow V\left(U^{\prime}\right)$ from the set $\pi^{-}(N)$ of directed paths in $\pi(N)$ that end in a tree vertex to the vertex set of $U^{\prime}$. Thus, the restriction $\psi_{N}=\left.\Psi_{N}\right|_{\pi_{0}(N)}$ of $\Psi_{N}$ to $\pi_{0}(N)$ induces a bijection from $\pi_{0}(N)$ to $L\left(U^{\prime}\right)$. Putting $\phi_{N}(x)=\left\{l \in L\left(U^{\prime}\right): \psi_{N}^{-1}(l) \in \pi_{x}(N)\right\}$, for all $x \in X$, completes the construction of $\mathcal{U}(N)$.

\subsection{The fold-up of a MUL-tree}

To state this construction, we again require further concepts which we introduce next. Suppose $\mathcal{G}=(G, \gamma)$ is a pair consisting of a rooted connected pseudoDAG $G$ and a labelling map 
$\gamma: X \rightarrow \mathcal{P}(L(G))$. If $G$ contains a cut-arc $a$ then we denote by $G(a)$ the connected component that contains head( $a)$ in its vertex set when deleting $a$. If $G(a)$ is a rooted tree then we denote the MUL-tree induced by $G(a)$ by $\mathcal{G}(a)$. By abuse of terminology, we say that $\mathcal{G}(a)$ is a subMUL-tree of $\mathcal{G}$ even if $\mathcal{G}$ itself is not a MUL-tree.

Suppose for the following that $a$ is a cut-arc of $\mathcal{G}$ such that $\mathcal{G}(a)$ is a subMUL-tree of $\mathcal{G}$. We say that $\mathcal{G}(a)$ is inextendible if there exists a cut arc $a^{\prime}$ in $\mathcal{G}$ distinct from $a$ such that the subMUL-trees $\mathcal{G}(a)$ and $\mathcal{G}\left(a^{\prime}\right)$ are isomorphic. We denote by $S_{\mathcal{G}(a)}$ the set of all subMUL-trees of $\mathcal{G}$ that are isomorphic with $\mathcal{G}(a)$ (including $\mathcal{G}(a)$ itself). In case $\mathcal{G}(a)$ is inextendible then we say that $\mathcal{G}(a)$ is maximal inextendible if every inextendible subMUL-tree $\mathcal{H}$ of $\mathcal{G}$ that contains an element in $S_{\mathcal{G}(a)}$ as a subMUL-tree is contained in $S_{\mathcal{G}(a)}$. In other words, $\mathcal{G}(a)$ is maximal inextendible if there is no other inextendible subtree that properly contains a subMUL-tree that is isomorphic to $\mathcal{G}(a)$.

For $\mathcal{M}=(M, \mu)$ a MUL-tree on $X$, we define the fold-up $F(\mathcal{M})$ of $\mathcal{M}$ to be the $X$-network obtained by applying the following operation until no inextendible subMUL-tree remains in $\mathcal{M}$. First select a maximal inextendible subMUL-tree $\mathcal{M}^{\prime}$ from $\mathcal{M}$. Then subdivide the incoming arc of the root of each subMUL-tree in $S_{\mathcal{M}^{\prime}}$ with a new vertex. Next, identify all subdivision vertices introduced at this step and delete all but one subMUL-tree in $S_{\mathcal{M}^{\prime}}$ from $\mathcal{M}$ and also the incoming arcs of their roots. Finally, replace $\mathcal{M}$ by the resulting rooted labelled pseudoDAG and repeat the above process for a maximal inextendible subMUL-tree $\mathcal{M}^{\prime}$ of $\mathcal{M}$.

Note that upon completion, the resulting pseudoDAG $F(\mathcal{M})$ is always an $X$-network and that this network is independent of the order in which the maximal inextendible subMUL-trees have been processed [16]. However, $F(\mathcal{M})$ need not be a phylogenetic network on $X$ as it might have parallel arcs. We call a MUL-tree $\mathcal{M}$ sound if $F(\mathcal{M})$ is a phylogenetic network. Note that stable binary phylogenetic networks were characterized in [19, Theorem 1] in terms of a vertex property. Also note that sound MUL-trees were characterized in [19] as those MUL-trees which do not contain a pair of isomorphic subMUL-trees whose roots share a parent. So, for example, the MUL-tree depicted in Figure 2(ii) is sound.

To help illustrate the fold-up operation, consider the MUL-tree $\mathcal{M}$ pictured in Figure 2(ii). Then the rooted, connected pseudoDAG on $X=\{1,2,3\}$ depicted in Figure 2(iii) is $F(\mathcal{M})$. Clearly, $F(\mathcal{M})$ is a phylogenetic network on $X$ and the MUL-trees $\mathcal{U}(F(\mathcal{M}))$ and $\mathcal{M}$ are isomorphic. Note however that $\mathcal{M}$ is also the un-fold of the $X$-network $N$ pictured in Figure 2 (i) and $F(\mathcal{M})$ and $N$ are clearly not isomorphic. We call an $X$-network $N$ that is isomorphic with $F(\mathcal{U}(N))$ stable. Note that for any stable phylogenetic network $N$ the un-fold $\mathcal{U}(N)$ of $N$ is a 
sound MUL-tree.

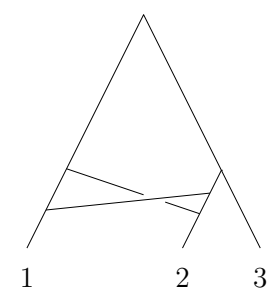

(i)

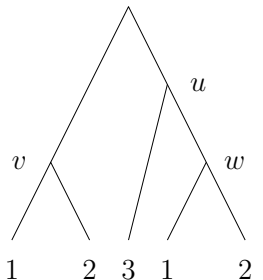

(ii)

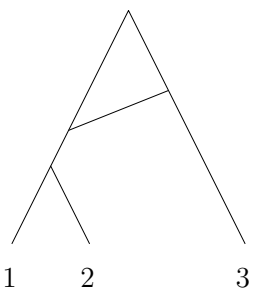

(iii)

Figure 2: (i) A phylogenetic network $N$ on $X=\{1,2,3\}$ that is not stable. (ii) The unfolded version $\mathcal{U}(N)$ of $N$ (ignoring interior labels for the moment). (iii) The folded version $F(\mathcal{U}(N))$ of $\mathcal{U}(N)$.

As we shall see, it is advantageous to describe the set of tree vertices of a folded-up MULtree $\mathcal{M}$ in terms of an equivalence relation $\sim$ on the vertex set of $\mathcal{M}$. To state that relation, suppose that $\mathcal{M}=(M, \mu)$ is a MUL-tree. Let $\sim$ denote the equivalence relation defined, for all $u, v \in V(M)$, by putting $u \sim v$ if $u=v$ or the subMUL-trees $\mathcal{M}_{v}$ and $\mathcal{M}_{u}$ of $\mathcal{M}$ rooted at $u$ and $v$, respectively, are isomorphic. Note that if $v$ and $w$ are two leaves of $\mathcal{M}$ then $v \sim w$ if and only if there exists some $x \in X$ such that $v, w \in \mu(x)$. We denote the quotient of $V(\mathcal{M})$ via $\sim$ by $V(\mathcal{M}) / \sim$, and we denote by $\bar{a}$ the equivalence class generated by a vertex $a \in V(\mathcal{M})$. We call the map $p_{\mathcal{M}}: V(\mathcal{M}) \rightarrow V(\mathcal{M}) / \sim$ defined by mapping each vertex of $M$ to its equivalence class under $\sim$ the projection of $\mathcal{M}$ to $V(\mathcal{M}) / \sim$. Note that if $u$ and $v$ are vertices in $\mathcal{M}$ such that $u$ is an ancestor of $v$, then $\left|p_{\mathcal{M}}(u)\right| \leq\left|p_{\mathcal{M}}(v)\right|$.

For example, for $v$ and $w$ the indicated interior vertices in the MUL-tree depicted in Figure1(ii), we have $w \sim v$. Furthermore, $|V(\mathcal{M}) / \sim|=10$ and four of those 10 equivalence classes are induced by the leaves of $\mathcal{M}$. Finally, $\left|p_{\mathcal{M}}(v)\right|=2$ and $\left|p_{\mathcal{M}}(u)\right|=1$.

As a first consequence, we have the following result whose straight-forward proof we leave to the interested reader.

Lemma 3.1. Suppose that $\mathcal{M}=(M, \mu)$ is a sound $M U L$-tree and that $u, v \in V(M)$. Then, $u \sim v$ if and only if the directed paths $\Psi_{F(\mathcal{M})}^{-1}(u)$ and $\Psi_{F(\mathcal{M})}^{-1}(v)$ in $F(\mathcal{M})$ have the same end vertex (which must necessarily be a tree vertex). In particular, if there exists a stable phylogenetic network $N$ such that $\mathcal{M}$ and $\mathcal{U}(N)$ are isomorphic then there exists a trivial bijection $\kappa_{N}$ : $V^{-}(N) \rightarrow V(\mathcal{U}(N)) / \sim$ from the set $V^{-}(N)$ of tree vertices of $N$ to the projection of $V(\mathcal{U}(N))$ to $V(\mathcal{U}(N)) / \sim$.

Note that Figure 2 indicates that the second part of Lemma 3.1 does not hold if we drop the assumption that $N$ is a stable phylogenetic network.

To be able to state an observation concerning stable phylogenetic networks which might be 
of interest in its own right, we require a further concept. Suppose $G=(V, E)$ and $G^{\prime}=\left(V^{\prime}, E^{\prime}\right)$ are two directed graphs. Then we define the union of $G$ and $G^{\prime}$ to be the directed graph with vertex set $V \cup V^{\prime}$ and arc set $E \cup E^{\prime}$. We call the union of two arc-disjoint directed paths $P$ and $P^{\prime}$ of $G$ which have the same start vertex and also the same end vertex a reticulation cycle of $G$.

Observation 3.2. Suppose that $N$ is a stable phylogenetic network and that $P_{1}, P_{2} \in \pi^{-}(N)$ distinct. Then the union of $P_{1}$ and $P_{2}$ contains a subgraph that is a reticulation cycle of $N$ if and only if the vertices $\Psi_{N}\left(P_{1}\right)$ and $\Psi_{N}\left(P_{2}\right)$ of $\mathcal{U}(N)$ are contained in two distinct but isomorphic subMUL-trees of $\mathcal{U}(N)$.

We again leave the straight-forward proof of the observation to the interested reader.

\section{Displaying and endorsing phylogenetic trees}

As suggested by Figure 1 any phylogenetic tree that is "contained" in a phylogenetic network $N$ is also "contained" (in possibly more than one way) in its associated MUL-tree $\mathcal{U}(N)$. However the converse need not hold. Understanding this relationship is the main purpose of this section.

We start with formalizing the idea of "containment" in an $X$-network and in a MUL-tree, respectively. Suppose for the following that $N$ is a $X$-network. If $N^{\prime}$ is a further $X$-network then we say that $N$ and $N^{\prime}$ are isomorphic if there exists a bijective map $f: V(N) \rightarrow V\left(N^{\prime}\right)$ that induces a graph isomorphism between $N$ and $N^{\prime}$ that maps every element of $X$ to itself. Following [19], we say that $N$ displays a phylogenetic tree $T$ on $X$ if there exists a subgraph $N^{\prime}$ of $N$ with leaf set $X$ that is isomorphic with a subdivision $T^{\prime}$ of $T$ and that isomorphism is the identity on $X$. Informally speaking, this means that $N^{\prime}$ can be obtained from $T$ by replacing $\operatorname{arcs}$ $a$ of $T$ by directed paths from $\operatorname{tail}(a)$ to head(a). It should however be noted that this definition does not imply that the root of a phylogenetic tree $T$ displayed by a phylogenetic network $N$ is also the root of $N$. Furthermore, it is worth noting that any phylogentic network gives rise to a phylogenetic tree that is displayed by it.

To illustrate the concept of displaying, consider the triplet $\tau=3 \mid 12$ and the phylogenetic network $N$ depicted in Figure 2(iii). Then $\tau$ is displayed by $N$ in two different ways one of which uses the root $\rho_{N}$ of $N$ as the root of $\tau$ and the other uses a child of $\rho_{N}$ as the root of $\tau$.

Suppose that $\mathcal{M}=(M, \mu)$ is a MUL-tree on $X$ where $|X| \geq 1$. Then we call a set $C \subseteq L(M)$ an $X$-set (of $\mathcal{M}$ ) if $|C \cap \mu(x)|=1$ holds for all $x \in X$. Informally speaking, an $X$-set for $\mathcal{M}$ contains for all $x \in X$ exactly one leaf of $M$ labelled by $x$. Note that if $|X| \geq 3$ then any $X$-set $C$ of $\mathcal{M}$ induces a phylogenetic tree $M_{C}$ on $X$ by first constructing a tree $M_{C}^{+}$from $M$ that is spanned by all leaves in $C$ and then, to obtain $M_{C}$, suppressing all resulting vertices of indegree 
and outdegree one - see again Figure 3 for an illustration of this construction. More formally, $V\left(M_{C}^{+}\right)$is the set of all $v \in V(\mathcal{M})$ that lie on a directed path from $l c a_{M}(C)$ to a leaf in $C$ and an $\operatorname{arc} a \in A(\mathcal{M})$ is an arc of $M_{C}^{+}$if there exists a leaf $l \in C$ such that $a$ is crossed by a directed path from $l c a_{M}(C)$ to $l$.

Note that for any phylogenetic tree $T$ displayed by a phylogenetic network $N$, there exists an $X$-set $C$ of $\mathcal{M}=\mathcal{U}(N)$ such that $T$ and $M_{C}$ are isomorphic. However, unless the vertices of $T^{\prime}$ corresponding to hybrid vertices of $N$ are suppressed, the subdivision $T^{\prime}$ of $T$ referred to in the definition of displaying is in general not isomorphic with $M_{C}^{+}$. Note that in case they are suppressed, this isomorphism can be defined in such a way that it is the identity on the leaf sets - see, for example, Figure 3

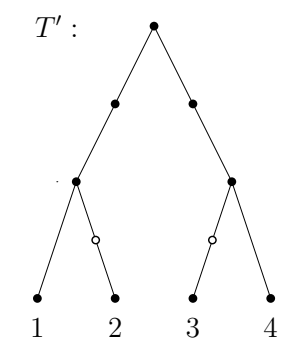

(i)

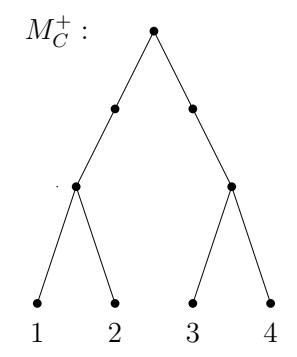

(ii)

Figure 3: (i) For the tree $T$ displayed by $N$ in terms of the dashed arcs in Figure 1(i), we depict the subdivision $T^{\prime}$ of $T$ that is isomorphic to a subgraph of $N$. The vertices corresponding to non-leaf tree-vertices (resp. hybrid vertices) of $N$ are indicated by black (resp. empty) dots. (ii) For $C$ the leaves of $\mathcal{M}$ that are incident with a dashed arc in Figure 1(ii), the tree $M_{C}^{+}$endorsed by $\mathcal{M}$. Vertices in $M_{C}^{+}$that correspond to non-leaf vertices of $\mathcal{M}$ are marked with black dots - see text for details.

We say that a phylogenetic tree $T$ is endorsed by a $M U L$-tree $\mathcal{M}=(M, \mu)$ via some $X$-set $C \subseteq L(M)$ if $T$ and $M_{C}$ are isomorphic. In case, the $X$-set $C$ is clear from the context, then we simply say that $T$ is endorsed by $\mathcal{M}$. Note that in [19] a phylogenetic tree that is endorsed by a MUL-tree $\mathcal{M}$ was said to be weakly displayed by the fold-up of $\mathcal{M}$.

To illustrate this definition, consider the stable phylogenetic network $N$ on $X=\{1,2,3,4\}$ depicted in bold lines in Figure 1(i). Then the dotted phylogenetic tree $T$ in Figure 1(ii) is endorsed by $\mathcal{U}(N)$. However $T$ is not displayed by $N$ since the joint grandparent of the leaves 2 and 3 in $N$ is used by the path from the root $\rho_{T}$ of $T$ to the leaf labelled 2 and also by the path from $\rho_{T}$ to the leaf labelled 3. Intriguingly, the phylogenetic tree in dashed lines in (ii) is endorsed by $\mathcal{U}(N)$ and is not only displayed by $N$ but also is a base tree for $N$ (see Theorem 5.1 for more on this). 
Note that the process of constructing the phylogenetic tree $M_{C}$ from a phylogenetic tree on $X$ endorsed by a MUL-tree $\mathcal{M}=(M, \mu)$ via some $X$-set $C$ trivially induces an ancestorrelationship-preserving injective map

$$
\xi_{C}: V\left(M_{C}\right) \rightarrow V(\mathcal{M})
$$

such that $\xi_{C}(x)$ is the unique element in $C \cap \mu(x)$, for all $x \in X$. Let $\xi_{C}^{+}: V\left(M_{C}^{+}\right) \rightarrow V(\mathcal{M})$ denote the canonical extension of $\xi_{C}$ to $V\left(M_{C}^{+}\right)$.

The following result is implied by [19, Theorem 6] which characterizes phylogenetic trees that are weakly displayed by a phylogenetic network. As such, it presents a first link between displaying a phylogenetic tree by a network and endorsing it by the un-fold of the network. To state it, we require a further map which we define next.

Suppose that $N$ is a phylogenetic network on $X$ and $C$ is an $X$-set of $\mathcal{M}=\mathcal{U}(N)$. Then for any tree $T^{\prime}$ obtained from $M_{C}^{+}$by suppressing some (but not necessarily all!) of its vertices of indegree and outdegree one it follows that there exists an injective map $P_{T^{\prime}}: V\left(T^{\prime}\right) \rightarrow \pi^{-}(N)$ which associates to each vertex $v$ of $T^{\prime}$ a (necessarily unique) directed path $P_{T^{\prime}}(v) \in \pi^{-}(N)$. Note that in case $M_{C}$ is displayed by $N$ there exists a subgraph $N^{\prime}$ of $N$ that is a subdivision of $M_{C}$ such that, for all vertices $v \in V\left(T^{\prime}\right)$, the directed path $P_{T^{\prime}}(v)$ does not cross any arc of $N$ that is not also contained in $N^{\prime}$.

Lemma 4.1. Suppose that $N$ is a phylogenetic network on $X$ and that $T$ is a phylogenetic tree on $X$ displayed by $N$. Then $T$ is endorsed by $\mathcal{U}(N)$ via some $X$-set $C$ of $\mathcal{U}(N)$. Moreover, $C$ can be chosen in such a way that $\Psi_{N} \circ P_{T}=\xi_{C}$.

Note that it is not difficult to see that the converse of Lemma 4.1 need not hold.

To be able to establish Theorem 4.2 which is the main result of this section, we next introduce two crucial maps. Suppose that $N$ is a phylogenetic network on $X$ and $T$ is a phylogenetic tree on $X$ that is displayed by $N$. Let $C$ be an $X$-set of $\mathcal{M}=\mathcal{U}(N)$ such that $T$ is endorsed by $\mathcal{M}$ via $C$. Then we put

$$
\overline{\xi_{C}}: V\left(M_{C}\right) \rightarrow V(\mathcal{M}) / \sim ; \quad v \mapsto p_{\mathcal{M}} \circ \xi_{C}(v)
$$

and

$$
\overline{\xi_{C}^{+}}: V\left(M_{C}^{+}\right) \rightarrow V(\mathcal{M}) / \sim ; \quad v \mapsto p_{\mathcal{M}} \circ \xi_{C}^{+}(v) .
$$

Note that the latter map was already mentioned in the introduction and is central for the remainder of the paper.

In addition, we denote by end $: \pi^{-}(N) \rightarrow V^{-}(N)$ a map that associates to a path $P \in \pi^{-}(N)$ the tree vertex of $N$ in which $P$ ends. Also, we denote by $f$ the map endo $\Psi_{N}^{-1}: V(\mathcal{M}) \rightarrow V^{-}(N)$ 
and by $\iota_{1}: V\left(M_{C}\right) \rightarrow V\left(M_{C}^{+}\right)$the map that maps every vertex in $V\left(M_{C}\right)$ to itself.

For $N$ a stable phylogenetic network on $X$ and $T$ a phylogenetic tree on $X$ that is displayed by $N$, we next summarize the main maps considered in this paper in Figure 4 for the convenience of the reader. Note that, by Lemma 4.1. $T$ is also endorsed by $\mathcal{U}(N)$ and that the $X$-set $C \subseteq L(\mathcal{U}(N))$ is chosen in such a way that $T$ and $M_{C}$ are isomorphic and that $\Psi_{N} \circ P_{T}=\xi_{C}$.

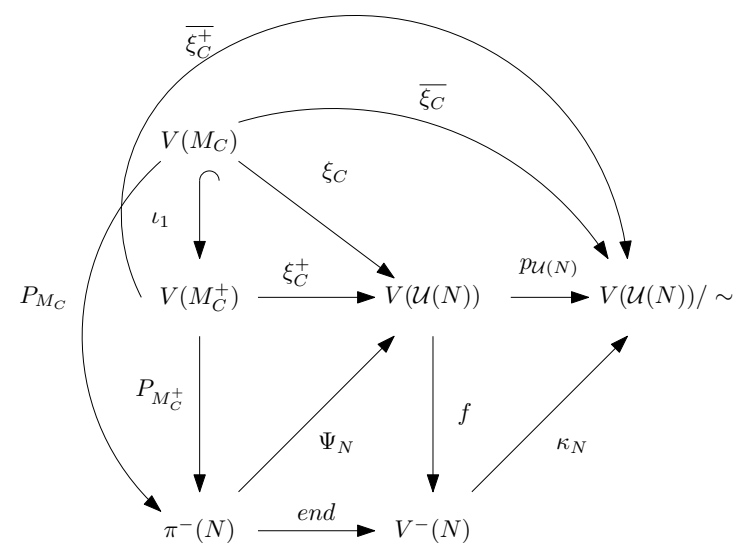

Figure 4: For $N$ a stable phylogenetic network on $X$ and $C$ an $X$-set of $\mathcal{U}(N)$ such that $M_{C}$ is displayed by $N$, we depict the main maps that play a role in this paper in the form of a commutative diagram. Note that since $N$ is stable, the phylogenetic networks $F(\mathcal{U}(N))$ and $N$ are isomorphic.

Armed with these definitions we are now ready to state the main result of this section. Informally speaking, it ensures that a phylogenetic tree $T$ is displayed by a stable phylogenetic network $N$ if and only if $T$ can be embedded (in the graph-theoretical sense) by $\overline{\xi_{C}^{+}}$into $\mathcal{U}(N)$ so that it is endorsed by $\mathcal{U}(N)$ via some subset $C \subseteq L(\mathcal{U}(N))$.

Theorem 4.2. Suppose that $N$ is a stable phylogenetic network on $X$ and that $T$ is a phylogenetic tree on $X$. Then, $T$ is displayed by $N$ if and only if there exists an $X$-set $C$ of $\mathcal{U}(N)$ that endorses $T$ and the map $\overline{\xi_{C}^{+}}$is injective.

Proof. Note first that since $N$ is stable we may assume without loss of generality that $N$ is in fact the fold-up $F(\mathcal{M})$ of a sound MUL-tree $\mathcal{M}=(M, \mu)$ on $X$. In view of Lemma 3.1, we therefore have that the map $\kappa_{N}: V^{-}(N) \rightarrow V(\mathcal{M}) / \sim$ in Figure 4 is bijective. Also note that we may assume without loss of generality that $N$ is not a phylogenetic tree on $X$ as otherwise the theorem clearly holds.

Assume first that $T$ is displayed by $F(\mathcal{M})$. Then, by Lemma 4.1. $T$ is endorsed by $\mathcal{M}$ via some $X$-set $C$ of $\mathcal{M}$. Thus, $T$ and $M_{C}$ must be isomorphic. Without loss of generality, we may therefore assume that $T$ is in fact $M_{C}$. Furthermore and again by Lemma 4.1 we may assume that $C$ is such that $\Psi_{N} \circ P_{M_{C}}=\xi_{C}$.

To see that the map $\overline{\xi_{C}^{+}}$is injective assume that there exist $w, w^{\prime} \in V\left(M_{C}^{+}\right)$such that $\overline{\xi_{C}^{+}}(w)=$ $\overline{\xi_{C}^{+}}\left(w^{\prime}\right)$. Then $\xi_{C}^{+}(w) \sim \xi_{C}^{+}\left(w^{\prime}\right)$. By Lemma 3.1 combined with the assumption that $\Psi_{N}$ is bijective it follows that the directed paths $\Psi_{N}^{-1}\left(\xi_{C}^{+}(w)\right)$ and $\Psi_{N}^{-1}\left(\xi_{C}^{+}\left(w^{\prime}\right)\right)$ must end in the same 
tree vertex $v$ contained in $V^{-}(F(\mathcal{M}))=V^{-}(N)$. Since $w=e n d \circ P_{M_{C}^{+}}(w)=e n d \circ \Psi_{N}^{-1}\left(\xi_{C}^{+}(w)\right)=$ $v=e n d \circ \Psi_{N}^{-1}\left(\xi_{C}^{+}\left(w^{\prime}\right)\right)=e n d \circ P_{M_{C}^{+}}\left(w^{\prime}\right)=w^{\prime}$ it follows that $\overline{\xi_{C}^{+}}$is injective.

Conversely, assume that there exists an $X$-set $C$ of $\mathcal{M}$ that endorses $T$ and $\overline{\xi_{C}^{+}}$is injective. Then we may assume again without loss of generality that $T$ is in fact $M_{C}$. Since $\overline{\xi_{C}^{+}}$is injective and $\kappa_{N}: V^{-}(N) \rightarrow V(\mathcal{M}) / \sim$ is bijective, it follows that the map $f \circ \xi_{C}^{+}: V\left(M_{C}^{+}\right) \rightarrow V^{-}(N)$ is injective. Hence, any two distinct vertices in $V\left(M_{C}^{+}\right)$get mapped to two distinct tree vertices of $N$. Since $f \circ \xi_{C}^{+}$preserves the ancestor relationships in $M_{C}^{+}$it follows that $f \circ \xi_{C}^{+}$induces an embedding of $M_{C}^{+}$into $N$. Since $M_{C}^{+}$is a subdivision of $M_{C}$ it follows that $M_{C}$ is displayed by $N$.

As it turns out, the equivalence postulated in Theorem 4.2 does not hold in case $N$ is not stable. To illustrate this fact, consider the phylogenetic network $N$ depicted in Figure $2(\mathrm{i})$. Then $N$ is not stable as $F(\mathcal{U}(N))$ is the phylogenetic network pictured in Figure 2(iii). As is easy to check the triplet $\tau=23 \mid 1$ is displayed by $N$. Also there is a unique choice of the $X$-set $C$ of $\mathcal{U}(N)$ such that $\tau$ is endorsed by $\mathcal{U}(N)$ via $C$. However for that choice of $C$ the map $\overline{\bar{\xi}_{C}^{+}}$is not injective as $\kappa_{N}^{-1} \circ \overline{\xi_{C}^{+}}$maps the vertices $v$ and $w$ of $\tau$ indicated in $\mathcal{U}(N)$ to the unique parent of leaves 1 and 2 in $F(\mathcal{U}(N))$.

\section{Stable phylogenetic networks that are tree-based}

As was observed in e.g. [27, some (but not all!) phylogenetic networks can be thought of as phylogenetic trees to which additional arcs have been added carefully. Motivated by this observation, we next turn our attention to characterizing such networks in case they are stable. We start with introducing terminology from [23] which we present within our framework.

Suppose that $N$ is a phylogenetic network $N$ on $X$. Then we say that $N$ is tree-based if there exists a phylogenetic tree $T$ on $X$, called the base tree of $N$, such that $N$ can be obtained from $T$ by applying the following 3 -step process. First, subdivide some of the $\operatorname{arcs}$ of $T$ by adding new vertices to them to obtain a rooted tree whose leaf set is $X$. Next, join these subdivision vertices by adding arcs between them, ensuring that no parallel arcs or directed cycles are created. Finally, suppress all subdivision vertices in the resulting rooted DAG whose indegree and outdegree is one. Note that, in addition to this definition of tree-based, 23] also present an alternative equally attractive definition of a non-binary tree-based phylogenetic network.

Clearly, a tree-based phylogenetic network may have more than one base tree. Also, all base trees of a tree-based network $N$ are displayed by $N$, although the converse is not true in general. An example for this is furnished by the dashed phylogenetic tree $T$ on $\{1, \ldots, 4\}$ in Figure 1(i), which is clearly displayed by the phylogenetic network $N$ depicted in that figure. 
However, although $T$ is a base tree for $N$ it is easy to check that the embedding of $T$ given in terms of the dashed edges does not lend itself as the starting point for constructing $N$ from $T$ as described above (see, for example, [25] for a discussion on the relationship between a tree-based phylogenetic network and a phylogenetic tree displayed by a phylogenetic network). As is easy to check, if the vertex set of $T$ is mapped into the vertex set of $N$ such that all tree vertices of $N$ are used then $T$ is not only displayed by $N$ but also is a base tree of $N$. To demonstrate that this relationship is not a coincidence is the purpose of the next result. To be able to establish it, we say that a phylogenetic network $N$ is compressed if it does not contain an arc $a$ such that $\operatorname{tail}(a)$ and $h e a d(a)$ are both hybrid vertices.

Theorem 5.1. Let $N$ be a stable phylogenetic network on $X$ and let $T$ be a phylogenetic tree on $X$. Then $T$ is a base tree for $N$ if and only if there exists an $X$-set $C$ of $\mathcal{U}(N)$ that endorses $T$ and the map $\overline{\xi_{C}^{+}}$is bijective.

Proof. Without loss of generality, we may assume that $N$ is not a phylogenetic tree as otherwise the result is trivial. Since $N$ is stable, we may assume without loss of generality that there exists a sound MUL-tree $\mathcal{M}=(M, \mu)$ such that $\mathcal{M}=\mathcal{U}(N)$. Moreover the map $\kappa_{N}: V^{-}(N) \rightarrow$ $V(\mathcal{M}) / \sim$ must be bijective.

Assume first that there exists an $X$-set $C$ of $\mathcal{M}$ such that $T$ is endorsed by $\mathcal{M}$ via $C$ and that $\overline{\xi_{C}^{+}}$is bijective. Then we may assume without loss of generality that $T$ is in fact $M_{C}$. Suppose for contradiction that $M_{C}$ is not a base tree of $N$. Then there exists a vertex $v$ in $N$ that is neither contained in $V\left(M_{C}\right)$ nor obtained from $M_{C}$ by the 3-step process described above. Since $M_{C}$ is displayed by $N$ in view of Theorem 4.2 and $f \circ \xi_{C}^{+}: V\left(M_{C}^{+}\right) \rightarrow V^{-}(N)$ is bijective as both $\kappa_{N}$ and $\overline{\xi_{C}^{+}}$are bijective, it follows that $v$ must be a hybrid vertex of $N$. Since $N$ is stable and therefore compressed by [19, Theorem 1], it follows that the unique child $c \in V(N)$ of $v$ as well as all parents of $v$ must be tree vertices of $N$. Let $p \in V(N)$ denote a parent of $v$. Then the bijectivity of $f \circ \xi_{C}^{+}: V\left(M_{C}^{+}\right) \rightarrow V^{-}(N)$ ensures that $p$ and $c$ are mapped to distinct vertices in $M_{C}^{+}$under $\left(f \circ \xi_{C}^{+}\right)^{-1}$. Note that since the map $\left(f \circ \xi_{C}^{+}\right)^{-1}$ is ancestor-preserving, $c^{\prime \prime}=\left(f \circ \xi_{C}^{+}\right)^{-1}(c)$ must be strictly below $p^{\prime \prime}=\left(f \circ \xi_{C}^{+}\right)^{-1}(p)$. Hence, $c^{\prime \prime}$ and $p^{\prime \prime}$ must either be vertices of $M_{C}$ or are subdivision vertices added to $M_{C}$ in the aforementioned 3-step process. Since $M_{C}$ is displayed by $N$ in view of Theorem 4.2 , it follows that $p^{\prime \prime}$ to $c^{\prime \prime}$ must also be vertices of $N$, and that the directed path from $p^{\prime \prime}$ to $c^{\prime \prime}$ in $M_{C}^{+}$induces a directed path from $p^{\prime \prime}$ to $c^{\prime \prime}$ in $N$ that must cross $v$. Hence, $T$ must be a base tree for $N$ which is impossible.

Conversely, assume that $T$ is a support tree for $N$. Then $T$ is displayed by $N$. By Theorem 4.2 , it follows that there exists an $X$-set $C$ of $\mathcal{M}$ such that $T$ is endorsed by $\mathcal{M}$ via $C$ and $\xi_{C}^{+}$is injective. It therefore remains to show that $\overline{\xi_{C}^{+}}$is surjective. Since we may assume without loss of generality that $T$ is in fact $M_{C}$ as $T$ is endorsed by $\mathcal{M}$ via $C$, it suffices to show that the map end $\circ \Psi_{N}^{-1} \circ \xi_{C}^{+}: V\left(M_{C}^{+}\right) \rightarrow V^{-}(N)$ is surjective. Suppose that $v \in V^{-}(N)$. Then $v$ is a tree vertex of $N$. Let $x \in X$ and let $P_{v}$ be the directed subpath of $P_{M_{C}}(x)$ starting at $\rho_{N}$ and ending in $v$. Since $\Psi_{N}$ preserves the ancestor relationships, it follows that, in $\mathcal{M}$, the vertex $\Psi_{N}\left(P_{v}\right)$ lies on the directed path from $\rho_{M}$ to the vertex $\Psi_{N}\left(P_{M_{C}}(x)\right)$. Since $M_{C}$ is a base tree for $N$, there exists some $u \in V\left(M_{C}^{+}\right)$such that $\xi_{C}^{+}(u)=\Psi_{N}\left(P_{v}\right)$. Hence, $\Psi_{N}^{-1} \circ \xi_{C}^{+}(u)=P_{v}$. Since $v$ is the end vertex of $P_{v}$ it follows that $e n d \circ \Psi_{N}^{-1} \circ \xi_{C}^{+}(u)=v$. Thus, end $\circ \Psi_{N}^{-1} \circ \xi_{C}^{+}$is surjective, as required. 
As in the case of Theorem 4.2 the equivalence postulated by Theorem 5.1 does not hold in case the phylogenetic network $N$ is not stable. Consider again the phylogenetic networks $N$ and $N^{\prime}=F(\mathcal{U}(N))$ depicted in Figure 2(i) and (iii) respectively. Then the triplet $23 \mid 1$ is a base tree for $N$ but not for $N^{\prime}$. Hence, by Theorem 5.1. there exists no $X$-set $C$ of $\mathcal{U}\left(N^{\prime}\right)$ that endorses that triplet and $\overline{\xi_{C}^{+}}$is bijective.

\section{Stable phylogenetic networks that are tree-child or reticulation-visible}

In this section, we turn our attention to clarifying the relationship between various key concepts originally introduced for binary phylogenetic networks. Our particular focus lies on the notion of a tree-child phylogenetic network [5] which has attracted a considerable amount of attention in the literature and also reticulation-visible networks [22. These concepts are closely related in that a phylogenetic network is reticulation-visible whenever it is tree-child (see e.g. 27] in case the network in question is binary). As an immediate consequence of the main result in this section (Theorem 6.4, we see that for stable phylogenetic networks both properties are also captured by our map $\overline{\xi_{C}^{+}}$.

We start with presenting formal definitions for both concepts. Let $N$ be a phylogenetic network on $X$. We say that an interior vertex $v$ of $N$ is tree-child if $v$ has a child $u$ that is a tree vertex. More generally, we say that $N$ is tree-child if all interior vertices of $N$ are tree-child. As an immediate consequence of [19, Corollary 1] it follows that any binary phylogenetic network that is tree-child must also be stable. It should however be noted that the converse is not true in general (see e.g. the network depicted in Figure 1(i) which is stable but not tree-child).

We show next that any tree-child network can also be seen as a stable phylogenetic network with an extra property in terms of displaying a phylogenetic tree. To do this, we require a further concept which we introduce next. Suppose $N$ is a phylogenetic network on $X$ and $w \in V^{\circ}(N)$. Then we say that $w$ is a vertex-stable ancestor of a leaf $x \in X$ if $w$ belongs to all directed paths from the root $\rho_{N}$ of $N$ to $x$. Note that vertex-stable ancestors were called "stable" ancestors in 22] and that a hybrid vertex that is a vertex-stable ancestors of some leaf is called "visible" in [22]. Also note that the root of a phylogenetic network $N$ is a vertex-stable ancestor for each leaf of $N$.

The proof of the following result was outlined in [27, page 249]. We include it for completeness sake as our notion of a phylogenetic network is slightly different from the one in [27].

Lemma 6.1. Let $N$ be a phylogenetic network on $X$. Then $N$ is tree-child if and only if for all vertices $v \in V^{\circ}(N)$, there exists a leaf $x_{v} \in X$ such that $v$ is a vertex-stable ancestor for $x_{v}$. 
Equivalently, $N$ is tree-child if and only if $N$ is compressed and, for all tree-vertices $v \in V^{\circ}(N)$, there exists a leaf $x_{v} \in X$ such that $v$ is a vertex-stable ancestor for $x_{v}$.

Proof. Assume first that $N$ is tree-child. Let $v \in V^{\circ}(N)$. Then there exists a directed path $P: v=v_{0}, v_{1}, \ldots, v_{k}$ of vertices of $N$ such that $v_{k}$ is a leaf and $v_{i}$ is a tree vertex, for all $0<i \leq k$. Hence, no vertex on $P$ other than possibly $v$ can be a hybrid vertex of $N$. Thus, $v$ must be a vertex-stable ancestor of $v_{k}$.

Conversely, assume that $N$ is not tree-child. We need to show that there exists an interior vertex of $N$ that is not a vertex-stable ancestor of any leaf of $N$. To see this note that since $N$ is not tree-child there must exist some vertex $v \in V^{\circ}(N)$ that is not tree-child. Hence, all children of $v$ must be hybrid vertices of $N$. Let $x$ be a leaf of $N$ that is below $v$. Then since a hybrid vertex of $N$ cannot be a leaf of $N$ there exists a child $w \in V^{\circ}(N)$ of $v$ that is an ancestor of $x$. Since $w$ must be a hybrid vertex by the choice of $v$, there must exist a directed path from the root $\rho_{N}$ of $N$ to $x$ that does not cross $v$. Since this argument applies to all leaves below $v$, it follows that there exists no leaf of $N$ for which $v$ is a vertex-stable ancestor, as required.

To see the second part of the lemma, it suffices to note that the (unique) child of a hybrid vertex in a compressed phylogenetic network must be a tree vertex. The stated characterization then follows from the straight-forward observation that a hybrid vertex $h$ is a vertex-stable ancestor of some leaf $x$ of $N$ if and only if the child of $h$ is a vertex-stable ancestor of $x$.

In a stable phylogenetic network, tree vertices that are vertex-stable ancestors for a given leaf can be characterized using the following proposition. To state it, we denote for an $X$-set $C$ of a MUL-tree $\mathcal{M}$ the last common ancestor of the elements of $C$ in $\mathcal{M}$ by $r_{C}$. Note that the pre-image of $r_{C}$ under $\xi_{C}^{+}$is the root of $M_{C}^{+}$. By abuse of terminology, we denote that root also by $r_{C}$. Also, for two non-empty sets $A$ and $B$ and a map $g: A \rightarrow B$, we denote by $g(A)$ the image of $A$ under $g$.

Proposition 6.2. Let $N$ be a stable phylogenetic network on $X$ and let $v$ be a tree vertex in $V^{\circ}(N)$. Then, there exists a leaf $x_{v}$ of $N$ such that $v$ is a vertex-stable ancestor of $x_{v}$ if and only if, for all $X$-sets $C$ of $\mathcal{M}=\mathcal{U}(N)$, one of the following two properties holds:

(i) $\kappa_{N}(v)$ belongs to the image set of $V\left(M_{C}^{+}\right)$under $\overline{\xi_{C}^{+}}$.

(ii) $v$ is an ancestor of $\kappa_{N}^{-1}\left(\overline{r_{C}}\right)$ in $N$.

Rather than continuing with the proof, we next illustrate Properties (i) and (ii) for the convenience of the reader. Consider the phylogenetic network $N^{\prime}$ and the MUL-tree $\mathcal{M}=\mathcal{U}\left(N^{\prime}\right)$ depicted in Figures 2(iii) and (ii) respectively. Let $\rho_{N^{\prime}}$ and $\rho_{\mathcal{M}}$ be the root of $N^{\prime}$ and $\mathcal{M}$ respectively. Clearly, $\rho_{N^{\prime}}$ is a vertex-stable ancestor of some leaf of $\mathcal{M}$. Consider now the four $X$-sets $C$ of $\mathcal{M}$. Three of these $X$-sets $C$ are such that $\rho_{M}=r_{C}$ and that, therefore, $\rho_{M}$ belongs to the vertex set of $M_{C}$. In this case $\overline{\xi_{C}^{+}}\left(\rho_{M}\right)$ coincides with $\kappa_{N^{\prime}}\left(\rho_{N^{\prime}}\right)$. For the fourth $X$-set $C$ i. e. the one comprising the three right-most leaves of $\mathcal{M}$, we have that $r_{C}=u$. Since $\kappa_{N^{\prime}}^{-1}(\bar{u})$ is a child of $\rho_{N^{\prime}}$ in $N^{\prime}$, it follows that $\rho_{N^{\prime}}$ satisfies Property (ii). 
Proof. Assume first that there exists a leaf $x_{v}$ of $N$ such that $v$ is a vertex-stable ancestor of $x_{v}$. Let $C$ be an $X$-set of $\mathcal{M}=(M, \mu)$. It suffices to show that if $v$ is not an ancestor of $\kappa_{N}^{-1}\left(\overline{r_{C}}\right)$ in $N$ then $\kappa_{N}(v) \in \overline{\xi_{C}^{+}}\left(V\left(M_{C}^{+}\right)\right)$.

Assume for contradiction that this is not the case, that is, $v$ is not an ancestor of $\kappa_{N}^{-1}\left(\overline{r_{C}}\right)$ in $N$ and $\kappa_{N}(v) \notin \overline{\xi_{C}^{+}}\left(V\left(M_{C}^{+}\right)\right)$. Then there exists no vertex $v^{\prime} \in V\left(M_{C}^{+}\right)$such that $v$ is contained in the equivalence class generated by $v^{\prime}$ under the projection $p_{\mathcal{M}}: V(\mathcal{M}) \rightarrow V(\mathcal{M}) / \sim$. Hence, there exists no element $x \in X$ and leaf $l \in C$ with $l \in \mu(x)$ such that the directed subpath of $P_{M_{C}^{+}}(l)$ of $N$ that starts at $f\left(r_{C}\right)$ and ends in $x$ contains $v$. Combined with the fact that, also by assumption, $v$ is not an ancestor of $\kappa_{N}^{-1}\left(\overline{r_{C}}\right)$ in $N$ it follows that there exists no element $x \in X$ and leaf $l \in C$ with $l \in \mu(x)$ such that $v$ is a vertex on the directed path $P_{M_{C}^{+}}(l)$. Consequently, for all $x \in X$, there exists a directed path in $\pi_{x}(N)$ that does not cross $v$. But then $v$ cannot be a vertex-stable ancestor of some element in $X$ which is impossible.

Conversely, assume that $v$ is not a vertex-stable ancestor of some element of $X$, that is, for every element $x \in X$, there exists a path $P_{x} \in \pi_{x}(N)$ that does not cross $v$. We need to show that there exists some $X$-set $C$ of $\mathcal{M}$ such that neither Property (i) nor Property (ii) of the proposition holds.

Put $C:=\bigcup_{x \in X}\left\{P_{x}\right\}$. Clearly $C$ is an $X$-set of $\mathcal{M}$. For a vertex $w \in V\left(M_{C}^{+}\right)$denote by $x_{w}$ a leaf of $M_{C}^{+}$below $w$. Since $P_{M_{C}^{+}}$is the map that assigns to a vertex $w \in V\left(M_{C}^{+}\right)$the subpath of $P_{x_{w}}$ in $N$ that starts at $\rho_{N}$ and ends in $w$ it follows that the end-vertex of $P_{M_{C}^{+}}\left(r_{C}\right)$ is $\kappa_{N}^{-1}\left(\overline{r_{C}}\right)$. Thus, $v$ is not an ancestor of $\kappa_{N}^{-1}\left(\overline{r_{C}}\right)$. So Property (ii) does not hold. Moreover, by construction, no path in $P_{M_{C}^{+}}\left(V\left(M_{C}^{+}\right)\right)$can contain $v$ as a vertex. Hence, there cannot exist some $v^{\prime} \in V\left(M_{C}^{+}\right)$such that $P_{M_{C}^{+}}\left(v^{\prime}\right)$ ends in $v$. But then there cannot exist some $v^{\prime} \in V\left(M_{C}^{+}\right)$ such that $\kappa_{N}(v)=\kappa_{N} \circ$ end $\circ P_{M_{C}^{+}}\left(v^{\prime}\right)=\overline{\xi_{C}^{+}}\left(v^{\prime}\right)$. Thus, $\kappa_{N}(v) \notin \overline{\xi_{C}^{+}}\left(V\left(M_{C}^{+}\right)\right)$Hence, Property (i) does not hold either. This concludes the proof.

To help illustrate the consequences of Proposition 6.2 we need to introduce further terminology. For $\mathcal{M}$ a MUL-tree and $C$ an $X$-set of $\mathcal{M}$, we denote by $V(\mathcal{M})^{C}$ the subset of $V(\mathcal{M})$ containing $r_{C}$ and all vertices $v$ of $\mathcal{M}$ such that no vertex $u \in V(\mathcal{M})$ below $v$ satisfies $u \sim r_{C}$. In other words, the set $V(\mathcal{M})^{C}$ is precisely the set of vertices $v$ of $\mathcal{M}$ such that the vertex $f\left(r_{C}\right)$ is not below the vertex $f(v)$, plus the vertex $r_{C}$ itself. Note that $V(\mathcal{M})^{C}=V(\mathcal{M})$ if and only if $r_{C}$ is the root of $\mathcal{M}$.

To illustrate this definition, consider the MUL-tree $\mathcal{M}$ depicted in Figure 2(ii), and the $X$-set $C$ of $\mathcal{M}$ that consists of the three leaves below the vertex labelled $u$. Then $r_{C}=u$ and $V(\mathcal{M})^{C}$ contains all vertices below $u$ and all vertices below $v$ because no vertex $v^{\prime} \in V(\mathcal{M})-\{v\}$ below $v$ satisfies $v^{\prime} \sim r_{C}$. However, the root $\rho_{\mathcal{M}}$ of $\mathcal{M}$ is not contained in $V(\mathcal{M})^{C}$, since $r_{C}$ is below $\rho_{\mathcal{M}}$ and $r_{C} \sim r_{C}$ clearly holds. Furthermore, for $\mathcal{M}$ the MUL-tree depicted in Figure 1(ii) and $C$ the $X$-set that consists of the leaves of the dashed tree in that figure, we have that $V(\mathcal{M})^{C}=V(\mathcal{M})$.

Armed with this notation and Proposition 6.2, we are now ready to characterize stable phylogenetic networks that are also tree-child or reticulation-visible in terms of our map $\overline{\xi_{C}^{+}}$. We begin with a straight-forward result that will turn out to be useful in this context. 
Lemma 6.3. Let $N$ be a stable phylogenetic network on $X$ and let $C$ be an $X$-set of $\mathcal{M}=\mathcal{U}(N)$. Then $\overline{\xi_{C}^{+}}\left(V\left(M_{C}^{+}\right)\right) \subseteq V(\mathcal{M})^{C} / \sim$.

Proof. Assume for contradiction that there exists a vertex $v \in V\left(M_{C}^{+}\right)$such that $\overline{\xi_{C}^{+}}(v) \notin$ $V(\mathcal{M})^{C} / \sim$. Then, the end vertex of the directed path $\Psi_{N}^{-1}\left(\xi_{C}^{+}\left(\rho_{C}\right)\right)$ is below the end vertex of the directed path $\Psi_{N}^{-1}\left(\xi_{C}^{+}(v)\right)$. But this is impossible since end $\circ \Psi_{N}^{-1} \circ \xi_{C}^{+}$must preserve the ancestor relationships in $M_{C}^{+}$as both the maps $\xi_{C}^{+}$and end $\circ \Psi_{N}^{-1}$ preserve them.

We are now ready to state the main result of this section which provides characterizations for when a stable phylogenetic network is tree-child or reticulation-visible. A phylogenetic network $N$ is called reticulation-visible if every hybrid vertex $h$ of $N$ is a vertex-stable ancestor of some leaf $x_{h}$ of $N$ [22] - see e. g. [1, 9] for more on such networks. Note that Lemma 6.1]implies that a tree-child network is also reticulation-visible. However note that, in general, a reticulation-visible network need neither be stable nor tree-child (see for example the phylogenetic network depicted in Figure 2(i)).

Theorem 6.4. Let $N$ be a stable phylogenetic network on $X$. We have:

(i) $N$ is tree-child if and only if for all $X$-sets $C$ of $\mathcal{M}=\mathcal{U}(N)$ the sets $\overline{\xi_{C}^{+}}\left(V\left(M_{C}^{+}\right)\right)$and $V(\mathcal{M})^{C} / \sim$ coincide.

(ii) $N$ is reticulation-visible if and only if for all $X$-sets $C$ of $\mathcal{M}=(M, \mu):=\mathcal{U}(N)$ and all non-root vertices $v$ of $\mathcal{M}$ for which $\left|p_{\mathcal{M}}(u)\right|<\left|p_{\mathcal{M}}(v)\right|$ holds for the parent $u$ of $v$, we have $p_{\mathcal{M}}(v) \in \overline{\xi_{C}^{+}}\left(V\left(M_{C}^{+}\right)\right)$.

Proof. (i): Without loss of generality we may assume that $N$ is not a phylogenetic tree. Then since $N$ is stable, $N$ must be compressed by [19, Theorem 1]. By the second part of Lemma 6.1, it follows that $N$ is tree-child if and only if for all tree-vertices $v \in V^{\circ}(N)$ there exists a leaf $x_{v} \in L(N)$ such that $v$ is a vertex-stable ancestor of $x_{v}$. In combination with Proposition 6.2, this implies that $N$ is tree-child if and only if for all $X$-sets $C$ of $\mathcal{M}$ and all tree vertices $v \in V^{\circ}(N)$, we either have that $v$ is an ancestor of $\kappa_{N}^{-1}\left(\overline{r_{C}}\right)$ in $N$ or that $\kappa_{N}(v)$ belongs to $A_{C}:=\overline{\xi_{C}^{+}}\left(V\left(M_{C}^{+}\right)\right)$.

Assume first that $N$ is tree-child. Let $C \subseteq L(\mathcal{M})$ denote an $X$-set of $\mathcal{M}$. In view of Lemma 6.3 it suffices to show that $V(\mathcal{M})^{C} / \sim$ is contained in $A_{C}$. Let $b \in V(\mathcal{M})^{C} / \sim$. Then there exists some $a \in V(\mathcal{M})^{C} \subseteq V(\mathcal{M})$ such that $p_{\mathcal{M}}(a)=\bar{a}=b$. Since $\Psi_{N}^{-1}(a) \in \pi^{-}(N)$ it follows that $a^{\prime}:=e n d \circ \Psi_{N}^{-1}(a)$ is a tree vertex of $N$.

If $r_{C} \neq a$ then the definition of $V(\mathcal{M})^{C}$ implies that $\kappa_{N}^{-1}\left(\overline{r_{C}}\right)=e n d \circ \Psi_{N}^{-1}\left(r_{C}\right)$ is not below $a^{\prime}$. Since $e n d \circ \Psi_{N}^{-1}$ is ancestor preserving, it follows that $a^{\prime}$ is not an ancestor of $\kappa_{N}^{-1}\left(\overline{r_{C}}\right)$. Combined with our observations at the beginning of this proof, it follows that $\kappa_{N}\left(a^{\prime}\right) \in A_{C}$ in case $a^{\prime}$ is not a leaf of $N$. Hence, there exists some $d \in V\left(M_{C}^{+}\right)$such that $b=\bar{a}=\kappa_{N} \circ$ end $\circ \Psi_{N}^{-1}(a)=$ $\kappa_{N}\left(a^{\prime}\right)=\overline{\xi_{C}^{+}}(d)$ in this case. If $a^{\prime}$ is a leaf of $N$ then $a^{\prime}$ is a leaf of $M_{C}^{+}$and, so, $\overline{\xi_{C}^{+}}\left(a^{\prime}\right)=\bar{a}=b$.

If $r_{C}=a$ then $b=\bar{a}=\overline{r_{C}}=\overline{\xi_{C}^{+}}\left(r_{C}\right)$. In summary, we obtain that $V(\mathcal{M})^{C} / \sim$ is contained in $A_{C}$ whenever $N$ is tree-child.

To see the converse implication it suffices to show that if $N$ is not tree-child then there must exist some $X$-set $C \subseteq L(\mathcal{M})$ such that $V(\mathcal{M})^{C} / \sim$ is not contained in $A_{C}$. So assume that $N$ is not tree-child. Then there must exists some tree vertex $w \in V^{\circ}(N)$ such that both its children $w_{1}$ and $w_{2}$ are hybrid vertices of $N$. Without loss of generality, we may assume that $w$ is as far away from the root $\rho_{N}$ of $N$ as possible. Note that $w_{1} \neq w_{2}$ as $N$ is a phylogenetic network and 
that $w$ is the only parent shared by $w_{1}$ and $w_{2}$ as $N$ is stable. For $i=1,2$, let $P_{i} \in \pi_{w_{i}}(N)$ denote a directed path form $\rho_{N}$ ending in $w_{i}$ that does not contain the arc $\left(w, w_{i}\right)$ (which must exist even if the other parent of $w_{i}$ is below $\left.w\right)$. Let $C \subseteq L(\mathcal{M})$ denote an $X$-set such that $M_{C}^{+}$ contains $P_{1}$ and $P_{2}$ in its vertex set. Let $P_{w} \in \pi_{w}(N)$ denote a directed path from $\rho_{N}$ to $w$. Then $P_{w} \in V(\mathcal{M})$ and the choice of $P_{1}$ and $P_{2}$ implies that $f\left(r_{C}\right)=$ end $\circ \Psi_{N}^{-1}\left(r_{C}\right)$ is not below $\underline{\text { end }} \circ \Psi_{N}^{-1}\left(P_{w}\right)=f\left(P_{w}\right)$ in $N$. By definition, it follows that $P_{w}$ is a vertex in $V(\mathcal{M})^{C}$. Hence, $\overline{P_{w}}$ is a vertex in $V(\mathcal{M})^{C} / \sim$. However, by construction, there cannot exist some $w^{\prime} \in V\left(M_{C}^{+}\right)$ such that $\xi_{C}^{+}\left(w^{\prime}\right)=P_{w}$ and, therefore, that $\overline{\xi_{C}^{+}}\left(w^{\prime}\right)=\overline{P_{w}}$. Thus, $V(\mathcal{M})^{C} / \sim$ is not contained in $A_{C}$, as required.

(ii): Note first that since $N$ is stable, and thus compressed by [19, Theorem 1], the (unique) child of a hybrid vertex of $N$ is a tree vertex. Hence, $N$ is reticulation-visible if and only if for all vertices $w$ of $N$ whose parent is a hybrid vertex, there exists a leaf $x_{w}$ of $N$ such that $w$ is a vertex-stable ancestor of $x_{w}$. Assume that $w$ is a tree vertex of $N$ whose parent $v$ is a hybrid vertex.

Assume first that $w$ is a vertex-stable ancestor of some leaf $x_{w}$ of $N$. Let $C$ denote an $X$-set of $\mathcal{M}$. Then, $\left(\xi_{C}^{+} \circ f\right)^{-1}(w)$ must be a vertex of $M_{C}^{+}$. Hence, $\overline{\xi_{C}^{+}} \circ\left(\xi_{C}^{+} \circ f\right)^{-1}(w)=$ $\kappa_{N}(w)$. Consequently, $p_{\mathcal{M}}\left(f^{-1}(w)\right)=\kappa_{N}(w) \in \overline{\xi_{C}^{+}}\left(V\left(M_{C}^{+}\right)\right)$. Let $w^{\prime} \in V(M)$ denote the parent of $f^{-1}(w)$ in $\mathcal{M}$ such that the directed path $P$ from $f\left(w^{\prime}\right)$ to $w$ in $N$ crosses $v$. To see that $\left|p_{\mathcal{M}}\left(w^{\prime}\right)\right|<\left|p_{\mathcal{M}}\left(f^{-1}(w)\right)\right|$, note first that $\left|p_{\mathcal{M}}\left(w^{\prime}\right)\right| \leq\left|p_{\mathcal{M}}\left(f^{-1}(w)\right)\right|$ must always hold. If $\left|p_{\mathcal{M}}\left(w^{\prime}\right)\right|=\left|p_{\mathcal{M}}\left(f^{-1}(w)\right)\right|$ held then $v$ cannot be a vertex on $P$ which is impossible.

Next, assume for contradiction that $w$ is not a vertex-stable ancestor of any leaf of $N$ but that for any $X$-set $C$ of $\mathcal{M}$ and all non-root vertices $v$ of $\mathcal{M}$ for which $\left|p_{\mathcal{M}}(u)\right|<\left|p_{\mathcal{M}}(v)\right|$ holds for the parent $u$ of $v$ we have $p_{\mathcal{M}}(v) \in \overline{\xi_{C}^{+}}\left(V\left(M_{C}^{+}\right)\right)$. Let $w_{1}$ and $w_{2}$ denote the two children of $w$. Then using the same notation as in the proof of the converse implication in Part (i), it follows that the image $\overline{P_{w}}$ of the directed path $P_{w}=f^{-1}(w)$ under the projection $p_{\mathcal{M}}$ must be a vertex in $V(\mathcal{M})^{C} / \sim$. Let $w^{\prime}$ be a vertex in $\mathcal{M}$ that is a parent of $w$ below $r_{C}$. Then $\left|p_{\mathcal{M}}\left(f^{-1}(w)\right)\right| \neq\left|p_{\mathcal{M}}\left(w^{\prime}\right)\right|$ must hold since the directed path from from $f\left(w^{\prime}\right)$ to $w$ must contain $v$. Since $\left|p_{\mathcal{M}}\left(w^{\prime}\right)\right| \leq\left|p_{\mathcal{M}}\left(f^{-1}(w)\right)\right|$ always holds, our assumption implies that $\kappa_{N}(w)=\overline{P_{w}}=p_{\mathcal{M}}\left(f^{-1}(w)\right)$ is contained in $\overline{\xi_{C}^{+}}\left(V\left(M_{C}^{+}\right)\right)$. In view of Proposition 6.2 it follows that $w$ is a vertex stable ancestor of some leaf of $N$ which is impossible.

As an immediate consequence of Theorem 6.4 we obtain the following result where we say that a phylogenetic tree $T$ on $X$ is strongly displayed by a phylogenetic network $N$ on $X$ if $T$ is displayed by $N$ and the root of $T$ is the root of $N$. We remark in passing that for binary phylogenetic networks this result was also shown as part of [25, Theorem 1.1].

Corollary 6.5. Let $N$ be a stable phylogenetic network on $X$ that is tree-child. Then, every phylogenetic tree on $X$ that is strongly displayed by $N$ is a base tree for $N$.

Proof. Let $T$ be a phylogenetic tree that is strongly displayed by $N$. Then $T$ is clearly displayed by $N$. Since $N$ is stable, Theorem 4.2 implies that there exists an $X$-set $C$ of $\mathcal{M}=(M, \mu):=$ $\mathcal{U}(N)$ such that $\overline{\xi_{C}^{+}}$is injective, $\xi_{C}^{+}\left(\rho_{T}\right)$ is the root of $\mathcal{M}$, and $T$ and $M_{C}$ are isomorphic. Hence, the sets $V(\mathcal{M})^{C} / \sim$ and $V(\mathcal{M}) / \sim$ coincide. Since $N$ is tree-child, Theorem 6.4 implies that the sets $\overline{\xi_{C}^{+}}\left(V\left(M_{C}^{+}\right)\right)$and $V(\mathcal{M})^{C} / \sim=V(\mathcal{M}) / \sim$ are also equal. Since $\overline{\xi_{C}^{+}}$is injective with image set $V(\mathcal{M}) / \sim$, it follows by Theorem 5.1 that $T$ is a base tree for $N$. 
Note that the converse of Corollary 6.5 is not true in general, even if the phylogenetic network $N$ considered is stable. For example, all phylogenetic trees strongly displayed by the stable phylogenetic network $N$ depicted in Figure 11i) are base trees for $N$, but $N$ is not tree-child. The reason for this is that if $N$ is a stable phylogenetic network on $X$ and $C$ is an $X$-set of $\mathcal{M}=(M, \mu):=\mathcal{U}(N)$ such that $\overline{\xi_{C}^{+}}$is injective but not bijective, then this does not imply that (although being isomorphic with $M_{C}$ ) $T$ is not a base tree for $N$. Indeed, there may exist a further $X$-set $C^{\prime}$ of $\mathcal{M}$ such that $T$ and $M_{C^{\prime}}$ are isomorphic and $\overline{\xi_{C^{\prime}}^{+}}$is bijective. By Theorem 5.1 . this implies that $M_{C^{\prime}}$ (and therefore also $T$ ) is a base tree for $N$.

\section{Some remarks about reconstructing stable phylogenetic networks}

In this section, we briefly turn our attention to the problem of constructing stable $X$-networks from induced substructures. For this, we focus on so called trinets and also certain subtrees of the MUL-tree obtained as the un-fold of a phylogenetic network. The reason for this is that in 17] it was pointed out that, in general, a binary phylogenetic network $N$ (and therefore also an $X$-network) is not encoded that is, up to equivalence, uniquely reconstructible from its induced set of triplets or set of displayed phylogenetic trees. Given the strong relationship between a phylogenetic network $N$ and its induced MUL-tree we start with subtrees induced by $\mathcal{U}(N)$.

Following [13, we call a MUL-tree on three leaves a MUL-triplet. So for example for $Y=$ $\{1,2\}$, the MUL-tree $(M, \mu)$ with leaf set $\{a, b, c\}$ and $l c a_{M}(a, b)$ strictly below $l c a_{M}(a, c)$ and $\mu(1)=\{a, c\}$ and $\mu(2)=\{b\}$ is a MUL-triplet on $Y$. Saying that a MUL-tree $\mathcal{M}$ displays a MUL-triplet $\tau$ if there exists a MUL-tree $\mathcal{M}^{\prime}$ obtainable from $\mathcal{M}$ by deleting vertices and arcs (suppressing resulting degree two vertices and, if this has rendered the root of $\mathcal{M}^{\prime}$ a vertex of degree one, identifying that root with its unique child) such that $\mathcal{M}^{\prime}$ and $\tau$ are isomorphic, it is easy to check that the MUL-triplet $(M, \mu)$ constructed in the previous example is displayed by the left MUL-tree depicted in Figure 5 (i) where we represent each leaf by its label.

Although it is straight-forward to check that this definition of displaying reduces to the one for phylogenetic trees it should be noted that although a phylogenetic tree is encoded by its set of displayed triplets a MUL-tree is in general not encoded by its set of displayed MUL-triplets. An example for this is furnished by the MUL-trees $\mathcal{M}$ and $\mathcal{M}^{\prime}$ on $X=\{1,2,3,4\}$ depicted in Figure 5 which display the same set of MUL-triplets (and this set has 21 elements). See also 15 . where similar observations were made for the set of splits (i.e. bipartitions) induced by the edges of an unrooted MUL-tree and [10] for the set of clusters (i. e. the set of leaves reachable from a vertex) in a MUL-tree. 


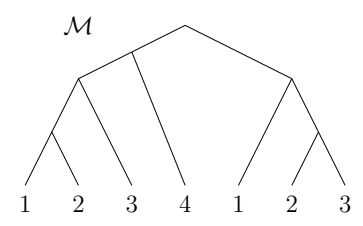

(i)

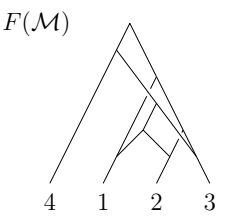

(ii)

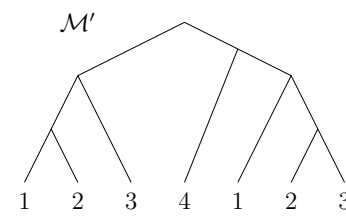

(iii)

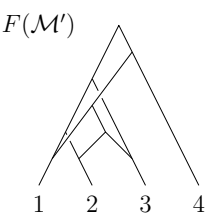

(iv)

Figure 5: Two MUL-trees $\mathcal{M}$ and $\mathcal{M}^{\prime}$ on $X=\{1,2,3,4\}$ displaying the same set of MUL-triplets on $X$. Note that the MUL-trees $\mathcal{M}_{Y}$ and $\mathcal{M}_{Y}^{\prime}$ are isomorphic, for all subsets $Y \subseteq X$ of size 3 . For ease of readability, we represent each leaf by its label.

Turning our attention towards the two non-isomorphic phylogenetic networks $F(\mathcal{M})$ and $F\left(\mathcal{M}^{\prime}\right)$ given in Figure 5(ii) and (iv), respectively, it is easy to check that they induce the same set of triplets on $X$. As a direct consequence of these observations it follows that, in general, stable phylogenetic networks are neither encoded by their set of induced triplets nor by the set of MUL-triplets induced by their un-folds.

We next turn our attention to understanding the encoding potential of trinets, that is, $X$ networks on three leaves [17]. Collections of trinets displayed by a tree-child network or so called level-2 networks are known to encode that network [28]. Since, in general, trinet systems do not encode phylogenetic networks [21] it is interesting to understand whether stable $X$-networks are encoded by the trinet system they display. To help shed light into this question, we require a certain leaf-removing operation for $X$-networks, which we now describe.

Let $N$ be an $X$-network, and let $l \in X$. Then the leaf-removing operation applied to $l$ consists of repeatedly applying the following three steps until a valid $(X \backslash\{l\})$-network is obtained:

(a) Remove the arc incident with $l$.

(b) Remove resulting hybrid vertices of outdegree zero (if any), and their incident arcs.

(c) Suppress resulting tree vertices of indegree and outdegree one (if any).

Now, let $Y$ be a subset of $X$ of size three or more, and let $N$ be an $Y$-network. Then we define the subnetwork $N_{Y}$ of $N$ induced by $Y$ to be the $Y$-network obtained from $N$ by applying the leaf-removing operations to all leaves of $N$ in $X \backslash Y$. If $Y$ is of size three, we say that a trinet $\tau$ on $Y$ is displayed by $N$ if $\tau$ and $N_{Y}$ are isomorphic. Note again that if $N$ is in fact a phylogenetic tree, then this definition of displaying reduces to the one for triplets.

By requiring in step (a) that all leaves of a MUL-tree $(M, \mu)$ contained in $\mu(l), l \in Y$, are removed, the aforementioned leaf-removing operation can also be extended to a leaf-removing 
operation for MUL-trees $\mathcal{M}$ to obtain a new MUL-tree $\mathcal{M}_{Y}$. In this case, we refer to $\mathcal{M}_{Y}$ as a sub-kMUL-tree of $\mathcal{M}$, where $k=|Y|$. Summarized in Observation 7.1, we have that the un-fold and leaf-removing operations are commutative:

Observation 7.1. Let $N$ be a phylogenetic network on $X$, and $Y$ a subset of $X$ of size three or more. Then the $M U L$-trees $\mathcal{U}\left(N_{Y}\right)$ and $\mathcal{U}(N)_{Y}$ are isomorphic.

Perhaps unsurprisingly, the equivalence in Observation 7.1 does not hold if the un-fold operation is replaced by the fold-up operation. The MUL-tree $\mathcal{M}$ on $X=\{1,2,3,4\}$ depicted in Figure 6(i) furnishes an example for this. For $Y=\{1,2,4\}$, we have that $F(\mathcal{M})_{Y}$ is the phylogenetic network depicted in Figure 6(ii), whereas $F\left(\mathcal{M}_{Y}\right)$ is the $Y$-network depicted in Figure6(iii).

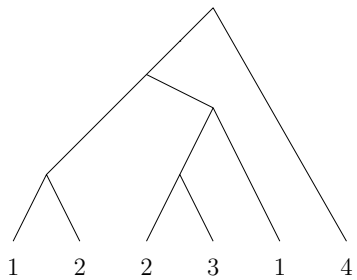

(i)

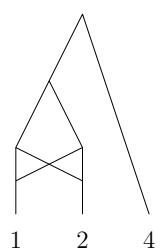

(ii)

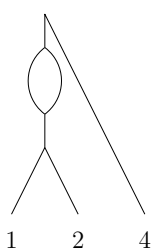

(iii)

Figure 6: (i) A MUL-tree $\mathcal{M}$ on $X=\{1,2,3,4\}$. (ii) The phylogenetic network on $Y=\{1,2,4\}$ obtained by first applying the fold-up operation to $\mathcal{M}$ and then the leaf-removing operation to the leaf labelled 3. (iii) The $Y$-network obtained by first applying the leaf-removing operation to the leaf labelled 3 and then applying the fold-up operation to the resulting MUL-tree.

Again, the MUL-trees $\mathcal{M}$ and $\mathcal{M}^{\prime}$ depicted in Figure 5 indicate that a MUL-tree is not, in general, encoded by the set of its displayed sub-3MUL-trees. Indeed, for all $Y \subset\{1,2,3,4\}$ of size three, the MUL-trees $\mathcal{M}_{Y}$ and $\mathcal{M}_{Y}^{\prime}$ are isomorphic. Note also that the example of these two MUL-trees implies that their respective fold-ups into the phylogenetic networks $F(\mathcal{M})$ and $F\left(\mathcal{M}^{\prime}\right)$ are not encoded by their sets of induced trinets.

\section{Discussion and open problems}

Stable phylogenetic networks constitute an interesting class of phylogenetic networks that have already proven useful for better understanding how polyploidy in, for example plants, has arisen. By investigating the popular tree-based, tree-child, and reticulation-visible properties for such networks we provide novel characterizations that shed light into the structural complexity of such networks. Although we only state our main results (i.e. Theorems 4.2 5.1 and 6.4 for stable phylogenetic networks, we remark in passing that they also apply to the more general $X$-networks (as long as the root of the network is not the tail of two parallel arcs in the case of 
Theorem 5.1]. Despite this, many open questions remain that might be of interest for future study. These include the following:

1. In their current form, stable phylogenetic networks are defined via a fold-up operation for MUL-trees. From an application point of view this is somewhat unsatisfactory as it is not always clear how to construct such a tree [15]. Thus it might be of interest to find combinatorial characterizations of stable phylogenetic networks that do not rely on that operation. Given our observations in Section 7 this will require alternative techniques as neither triplets, trinets, or general phylogenetic trees can be used for this.

2. How can we quantify how close two stable phylogenetic networks are. Given that we obtained in Theorem 5.1 a characterization for when a phylogenetic tree that is endorsed by a MUL-tree $\mathcal{M}$ is in fact a base tree of $F(\mathcal{M})$ and that tree-based networks are defined via base trees, it might be interesting to see if the similarity measures defined in [7] can be used for this.

\section{Acknowledgement}

The authors would like to thank the editor and the anonymous referee for their constructive comments and suggestions to improve the paper. GS would like to thank the University of East Anglia for hosting him during part of this work.

\section{References}

[1] Bordewich, M., Semple, C., 2016. Reticulation-visible networks. Advances in Applied Mathematics $78,114-141$.

[2] Bordewich, M., Semple, C., 2018. A universal tree-based network with the minimum number of reticulations. Discrete Applied Mathematics 250, 357-362.

[3] Bordewich, M., Semple, C., Tokac, N., 2018. Constructing tree-child networks from distance matrices. Algorithmica 80(8), 2240-2259.

[4] Brysting, A., Oxelman, B., Huber, K., Moulton, V., Brochmann, C., 2007. Untangling complex histories of genome mergings in high polyploids. Syst. Biol. 56(3), 467-476.

[5] Cardona, G., Rosselló, F., Valiente, G., 2007. Comparison of tree-child phylogenetic networks. IEEE/ACM Transactions on Computational Biology and Bioinformatics 6, 552-569. 
[6] Czabarka, E., Erdõs, P., Johnsona, V., Moulton, V., 2013. Generating functions for multilabeled trees. Discrete Applied Mathematics 161(1-2), 107-117.

[7] Francis, A., Semple, C., Steel, M., 2018. New characterisations of tree-based networks and proximity measures. Advances in Applied Mathematics 93, 93-107.

[8] Francis, A. R., Steel, M., 2015. Which phylogenetic networks are merely trees with additionals arcs? Syst. Biol. 64(5), 768-777.

[9] Gambette, P., Gunawan, A. D., Labarre, A., Vialette, S., Zhang, L., 2015. Locating a tree in a phylogenetic network in quadratic time. In: Przytycka, T. (Ed.), Research in Computational Molecular Biology (RECOMB 2015). Vol. 9029. Lecture Notes in Computer Science, pp. 96-107.

[10] Ganapathy, G., Goodson, B., Jansen, R., Le, H. S., Ramachandran, V., Warnow, T., 2006. Pattern identification in biogeography. IEEE/ACM Trans. Comput. Biol. Bioinform. 3(4), $334-46$.

[11] Gunawan, A. D. M., DasGupta, B., Zhang, L., 2017. A decomposition theorem and two algorithms for reticulation-visible networks. Information and Computation 252, 161-175.

[12] Gusfield, D., 2014. ReCombinatorics: the algorithmics of ancestral recombination graphs and explicit phylogenetic networks. Cambridge: MIT Press.

[13] Hassanzadeh, R., Eslahchi, C., Sung, W.-K., 2014. Do triplets have enough information to construct the multi-labeled phylogenetic tree? PLoS ONE 9(7), e103622.

[14] Hayamizu, M., 2016. On the existence of infinitely many universal tree-based networks. J. Theoret. Biol. 396, 204-206.

[15] Huber, K. T., Lott, M., Moulton, V., Spillner, A., 2008. The complexity of deriving multilabeled trees from bipartitions. Journal of Computational Biology 15(6), 639-651.

[16] Huber, K. T., Moulton, V., 2006. Phylogenetic networks from multi-labelled trees. Journal of Mathematical Biology 52(2), 613-32.

[17] Huber, K. T., Moulton, V., 2013. Encoding and constructing 1-nested phylogenetic networks with trinets. Algorithmica 66(3), 714-738. 
[18] Huber, K. T., Moulton, V., Spillner, A., Storandt, S., Suchecki, R., 2012. Computing a consensus of multilabeled trees. In: Bader, D. A., Mutzel, P. (Eds.), Proceedings of the Fourteenth Workshop on Algorithm Engineering \& Experiments (ALENEX 12). Vol. 5805. SIAM, p. 84-92.

[19] Huber, K. T., Moulton, V., Steel, M., Wu, T., 2016. Folding and unfolding phylogenetic trees and networks. Journal of Mathematical Biology 73(6-7), 1761-1780.

[20] Huber, K. T., Oxelman, B., Lott, M., Moulton, V., 2006. Reconstructing the evolutionary history of polyploids from multilabeled trees. Molecular Biology and Evolution 23, 17841791.

[21] Huber, K. T., van Iersel, L., Moulton, V., Wu, T., 2015. How much information is needed to infer reticulate evolutionary histories? Systematic Biology 64(1), 102-111.

[22] Huson, D., Rupp, R., Scornavacca, C., 2010. Phylogenetic Networks. Cambridge University Press.

[23] Jetten, L., van Iersel, L., 2018. Nonbinary tree-based phylogenetic networks. IEEE/ACM Trans. Comput. Biol. Bioinform. 15(1), 205-217.

[24] Lott, M., Spillner, A., Huber, K. T., Petri, A., Oxelman, B., Moulton, V., 2009. Inferring polyploid phylogenies from multiply-labeled gene trees. BMC Evolutionary Biology 9, 216.

[25] Semple, C., 2016. Phylogenetic networks with every embedded phylogenetic tree a base tree. Bulletin of Mathematical Biology 78(1), 132-137.

[26] Semple, C., Steel, M., 2003. Phylogenetics. Oxford University Press.

[27] Steel, M., 2016. Phylogeny: Discrete and Random Processes in Evolution. SIAM.

[28] van Iersel, L. J. J., Moulton, V., 2014. Trinets encode tree-child and level-2 phylogenetic networks. Journal of Mathematical Biology 68(7), 1707-1729.

[29] van Iersel, L. J. J., Semple, C., Steel, M., 2010. Locating a tree in a phylogenetic network. Inform. Process. Lett. 110, 1037-1043.

[30] Wieseke, N., Bernt, M., Middendorf, M., 2013. Unifying parsimonious tree reconciliation. In: Darling, A., Stoye, J. (Eds.), International Workshop on Algorithms in Bioinformatics (WABI 2013) Algorithms in Bioinformatics. Vol. 126. Lecture Notes in Computer Science, pp. 200-214. 
[31] Zhang, L., 2016. On tree-based phylogenetic network. J. Comput. Biol. 23, 553-565. 